\title{
Incorporation of alternative amino acids into cyanophycin by different cyanophycin synthetases heterologously expressed in Corynebacterium glutamicum
}

\author{
Ramona Wördemann ${ }^{1} \mathbb{B}$, Lars Wiefel ${ }^{1}$, Volker F. Wendisch ${ }^{2}$ and Alexander Steinbüchel ${ }^{1,3^{*}}$
}

\begin{abstract}
Cyanophycin (multi--L-arginyl-poly-L-aspartic acid; also known as cyanophycin grana peptide [CGP]) is a biopolymer that could be used in various fields, for example, as a potential precursor for the synthesis of polyaspartic acid or for the production of CGP-derived dipeptides. To extend the applications of this polymer, it is therefore of interest to synthesize CGP with different compositions. A recent re-evaluation of the CGP synthesis in C. glutamicum has shown that $C$. glutamicum is a potentially interesting microorganism for CGP synthesis with a high content of alternative amino acids. This study shows that the amount of alternative amino acids can be increased by using mutants of C. glutamicum with altered amino acid biosynthesis. With the DM1729 mutant, the lysine content in the polymer could be increased up to $33.5 \mathrm{~mol} \%$. Furthermore, an ornithine content of up to $12.6 \mathrm{~mol} \%$ was achieved with ORN2 $\left(\mathrm{P}_{\text {gdh } 4}\right)$. How much water-soluble or insoluble CGP is synthesized is strongly related to the used cyanophycin synthetase. CphA $\mathrm{Dh}_{\mathrm{D}}$ synthesizes soluble CGP exclusively. However, soluble CGP could also be isolated from cells

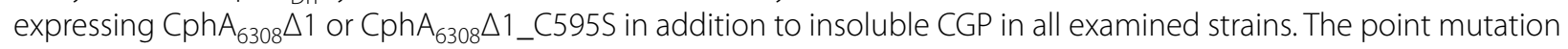
in $\mathrm{CphA}_{6308} \triangle 1$ 1_C595S partially resulted in a higher lysine content. In addition, the CGP content could be increased to $36 \%$ of the cell dry weight under optimizing growth conditions in C. glutamicum ATCC13032. All known alternative major amino acids for CGP synthesis (lysine, ornithine, citrulline, and glutamic acid) could be incorporated into CGP in C. glutamicum.
\end{abstract}

Keywords: Alternative amino acids, Corynebacterium glutamicum, Cyanophycin, Lysine, Ornithine

\section{Introduction}

The polyamide cyanophycin is typically composed of poly(aspartic acid) as a backbone to which arginine residues are attached; it is also referred to as multi-L-arginyl-poly-L-aspartic acid or as cyanophycin grana peptide (CGP). CGP is non-ribosomal polypeptide synthesised by cyanophycin synthetases (CphA, EC 6.3.2.29/EC

\footnotetext{
*Correspondence: steinbu@uni-muenster.de

${ }^{1}$ Institut für Molekulare Mikrobiologie und Biotechnologie (IMMB), Westfälische Wilhelms-Universität Münster, Corrensstraße 3, 48149 Münster, Germany

Full list of author information is available at the end of the article
}

6.3.2.30). During heterologous production of CGP, other alternative amino acids can also be incorporated into the polymer in addition to aspartic acid and arginine. Instead of arginine, larger amounts of lysine, citrulline or ornithine and several others were also incorporated (Berg et al. 2000; Steinle et al. 2009; Wiefel et al. 2011). Glutamic acid can also be incorporated into the backbone as an alternative to aspartic acid (Wiefel et al. 2019a and references cited therein). In addition, biochemically incorporated constituents can be modified enzymatically or chemically (Frommeyer et al. 2014; Wiefel and Steinbüchel 2016, Wiefel 2019b). These variations of the polymer can change the solubility behavior of the polymer (Wiefel 
and Steinbüchel 2014). Variations in CGP are especially interesting to extend the possible field of applications of CGP. For example, CGP-derived dipeptides can be used as food supplements or animal feed additives or may have medical and cosmetic applications (Sallam and Steinbüchel 2010). The dipeptides are produced by enzymatic digestion of CGP employing cyanophycinase (intracellular CphB or extracellular CphE; EC 3.4.15.6), usually producing Asp-Arg dipeptides and related oligopeptides (Sallam et al. 2009).

Corynebacterium glutamicum in particular could be an interesting candidate for the production of CGP with a high content of alternative amino acids, as this bacterium is traditionally used for the production of amino acids on an industrial scale (Wendisch 2020). C. glutamicum is best known for the production of glutamate and lysine, both of which can be used for the synthesis of CGP. However, the other amino acids, which can be used for the synthesis of CGP, can also be produced in larger quantities by C. glutamicum. On the other hand, the thick and rigid cell wall of C. glutamicum is disadvantageous for isolating CGP from the cells in comparison to other microorganisms (Wiefel et al. 2019a).

In the early 2000s, CGP synthesis in C. glutamicum was studied for the first time. At that time only a CGP yield of less than 3\% of the dry cell mass was obtained from $C$. glutamicum cells (Aboulmagd et al. 2001b). At that time the existence of water-soluble CGP, which usually contains a high proportion of lysine, was not known, yet. For this reason, research on this topic was recently resumed. During this re-evaluation by Wiefel et al. (2019a), different vectors, CphAs and media were examined. It was shown that the strains can accumulate up to $17 \%$ of their cells dry mass CGP. It was also shown for the first time that glutamic acid can replace aspartic acid in the backbone of CGP (Wiefel et al. 2019a). The aim of this study was to improve the CGP synthesis in C. glutamicum with regard to the incorporation of alternative amino acids into the polymer. For this, optimized cultivation conditions and modified strains of C. glutamicum were used to try to increase the proportion of alternative amino acids and to further increase the CGP yield. Two variants of CphA from Synechocystis sp. PCC6308 and CphA from $D$. hafniense DSM 10664 were used for the investigations. Besides the wild type, mutants of C. glutamicum were used, which produce increased amounts of lysine, ornithine or cadaverine.

\section{Materials and methods}

Bacterial strains, media, and growth conditions

All bacterial stains and plasmids used in this study are listed in Table 1. E. coli strains were used for plasmid maintenance and propagation and were cultivated at $30^{\circ} \mathrm{C}$ and a stirring rate of $130 \mathrm{rpm}$ in lysogeny broth (LB) medium (Sambrook et al. 1989). Usually, $10 \mathrm{ml}$ medium in $100 \mathrm{ml}$ Erlenmeyer flask were used. For C. glutamicum strains BHIS (Tauch et al. 2002), CASO + G (Persicke et al. 2011) and CGXII (Keilhauer et al. 1993) medium were used. Strains harbouring plasmids were grown in presence of $25 \mu \mathrm{g}$ kanamycin per $\mathrm{ml}$. For the arginine auxotrophic ORN2 $\left(\mathrm{P}_{\text {gdh } 4}\right)$ strain (Jensen et al. 2015), $15 \mathrm{mM}$ arginine was added to the CGXII medium. To monitor cell growth, the optical density was measured at $600 \mathrm{~nm}\left(\mathrm{OD}_{600}\right)$. For cyanophycin synthesis in C. glutamicum two precultures with 10 and $50 \mathrm{ml}$ medium in 100 and $250 \mathrm{ml}$ Erlenmeyer flasks, respectively, were

Table 1 Strains and plasmids used in this study

\begin{tabular}{|c|c|c|}
\hline Strain or plasmid & Relevant characteristics & Reference or source \\
\hline \multicolumn{3}{|l|}{ Strains } \\
\hline E. coli TOP10 & $\begin{array}{l}\text { F- } m c r A \Delta(m r r-h s d \text { RMS-mcrBC) } \$ 80 l a c Z \Delta M 15 \Delta l a c X 74 \text { recA1 araD139 } \Delta \text { (araleu)7697 } \\
\text { ga/U gal } \\
\text { KrpsL }\left(\mathrm{Str}^{R}\right) \text { endA1 nupG }\end{array}$ & Invitrogen, Carlsbad, USA \\
\hline C. glutamicum ATCC 13,032 & Wild type, Biotin-auxotroph & DSMZ, Braunschweig, Germany \\
\hline C. glutamicum DM1729 & ATCC 13032 pyc $^{\text {P458S }}$, hom V59A, lys $^{\text {T3111 }}$ & Georgi et al. (2005) \\
\hline C. glutamicum ORN2 $\left(\mathrm{P}_{\text {gdh } 4}\right)$ & $\begin{array}{l}\text { ATCC } 13032 \text { with in-frame deletion of prophages CGP1 (cg1507-cg1524), CGP2 } \\
\text { (cg1746-cg1752), and CGP3 (cg1890-cg2071), } \triangle a r g F R G, \\
\text { P gdh4: - 35: TTGCCA - 10: TATAAT }\end{array}$ & Jensen et al. (2015) \\
\hline \multicolumn{3}{|l|}{ Plasmids } \\
\hline pVWEx1 & $\mathrm{P}_{\mathrm{tac}} \mid \mathrm{aCl}^{\mathrm{a}}, \mathrm{Km}^{\mathrm{R}}$ & Peters-Wendisch et al. (2001) \\
\hline pVWEx1::cphA $A_{6308} \Delta 1$ & pVWEx1 with $c p h A_{6308} \Delta 1$ (Pstl/Xbal) & Wiefel et al. (2019a) \\
\hline pVWEx1::cphA 6308 $\Delta 1$ C595S & pVWEx1 with cphA $A_{6308} \Delta 1 \_C 595 S($ Pstl/Xbal) & This study \\
\hline pVWEx1::cphA $A_{D h}$ & pVWEx1 with $c p h A_{D h}(S d a l / X b a l)$ & Wiefel et al. (2019a) \\
\hline pEC-XT99A::/dcC & 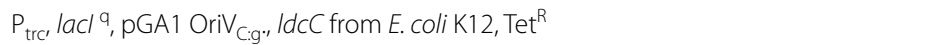 & Sgobba et al. (2018) \\
\hline
\end{tabular}

$\mathrm{Km}^{R}$ kanamycin resistance, $\operatorname{Tet}^{R}$ tetracycline resistance 
used to inoculate the main culture containing $100 \mathrm{ml}$ in a $500 \mathrm{ml}$ Erlenmeyer flask with two baffles. The first preculture with $\mathrm{CASO}+\mathrm{G}$ medium was inoculated with a cryoculture and was cultivated for about $8 \mathrm{~h}$. Before inoculating the second preculture, the cells were washed with washing buffer ( $50 \mathrm{mM}$ TRIS, $50 \mathrm{mM} \mathrm{NaCl}, \mathrm{pH}$ 6,3). For the second preculture, CGXII medium was used as the main culture. The main culture was inoculated by a $16-\mathrm{h}$ second preculture to an initial $\mathrm{OD}_{600}$ of 0.2 and induced after $4 \mathrm{~h}$ with $1 \mathrm{mM}$ IPTG. In contrast to the previous studies of Wiefel et al. (2019a) the strains were not cultivated under biotin limiting conditions.

\section{Cultivation of C. glutamicum strain DM1729 pVWEx1::cphA ${ }_{6308} \Delta 1_{-}$C595S in a stirred tank reactor}

A Biostat UD30 stainless steel reactor (B. Braun Biotech International, Melsungen, Germany) was used for batch cultivation of C. glutamicum strain DM1729 harboring pVWEx1::cph $A_{6308} \Delta 1_{-}$C595S in 20-liter scale. Two fermentations were performed. In the first fermentation normal CGXII medium according to Keilhauer 1993 was used, only without addition of MOPS buffer. For the second fermentation, $5 \mathrm{~g} / \mathrm{l}$ CASO broth (Carl Roth, Karlsruhe, Germany) was added to the medium instead of urea. The fermentations were inoculated with an $\mathrm{OD}_{600}$ between 0.7 and 0.8. An IPTG concentration of $0.1 \mathrm{mM}$ was used for induction. Cultivation was carried out at $30{ }^{\circ} \mathrm{C}$ and a $\mathrm{pO}_{2}$ saturation of $30 \%$ in the medium, which was controlled by stirring rates between 100 and $800 \mathrm{rpm}$ and aeration rates between 0.5 and $2.0 \mathrm{vvm}$ (volume per volume $\times$ minute). The $\mathrm{pH}$ was kept constant at a value of 7 with $4 \mathrm{M} \mathrm{NaOH}$ or $4 \mathrm{M} \mathrm{HCl}$. As antifoam agent Struktol $^{\circledR}$ J 673 A (Schill+Seilacher, Hamburg, Germany) was used. Samples with a volume of $100 \mathrm{ml}$ were taken to determine $\mathrm{OD}_{600}$, cells dry masses and CGP content of the cells.

\section{Molecular biology techniques}

Plasmid DNA from E. coli strains was isolated using the GenJET plasmid miniprep kit (Thermo Scientific, Waltham, USA). The plasmids contain the genes for the cyanophycin synthetases $\mathrm{CphA}_{6308} \Delta 1, \mathrm{CphA}_{6308} \Delta 1_{-}$ C595S or $\mathrm{CphA}_{\mathrm{Dh}}$ (see Table 2). Upstream of the genes a ribosome binding site (RBS) is located which was optimized for expression in C. glutamicum (GAA AGGAGGCCCTTCAG, Siebert and Wendisch 2015). For isolation of plasmids from C. glutamicum strains, $15 \mathrm{mg}$ lysozyme were added per $\mathrm{ml}$ to the resuspension buffer, and the resuspended cells were incubated for $2 \mathrm{~h}$ at $37{ }^{\circ} \mathrm{C}$ before following the protocol of the manufacturer (Thermo Scientific, Waltham, USA).

Ampicillin was added to the medium during the production of electrocompetent C. glutamicum cells (Jensen et al. 2015). Transformation of C. glutamicum was performed by electroporation and a heat-shock at $46{ }^{\circ} \mathrm{C}$ for 6 min (van der Rest et al. 1999). Restriction analyses were carried out for control purposes. The restriction enzymes were purchased from Thermo Scientific.

\section{Isolation of cyanophycin from C. glutamicum}

For isolation of CGP from C. glutamicum, cells were harvested by centrifugation $\left(20 \mathrm{~min}, 5000 \times g, 4{ }^{\circ} \mathrm{C}\right)$, washed with $0.9 \%(\mathrm{wt} / \mathrm{vol}) \mathrm{NaCl}$, and freeze-dried. After determining the cells dry mass, the cell disintegration of the cells was carried out using an ultrasonic disintegrator. For this purpose, the freeze-dried cells were crushed and resuspended in $\mathrm{H}_{2} \mathrm{O}(20 \mathrm{ml} / \mathrm{g})$. The suspension was then sonicated for $1 \mathrm{~min} / \mathrm{ml}$ with the UP200S ultrasonic processor and shaken overnight at $4{ }^{\circ} \mathrm{C}$. Insoluble material was sedimented by centrifugation ( $30 \mathrm{~min}, 5000 \times \mathrm{g}$ ), and the water-soluble CGP, which was contained in the supernatant, was precipitated using $1.5 \mathrm{vol}$ ice-cold ethanol. After centrifugation, the CGP pellet was washed with acetone and dried at $70{ }^{\circ} \mathrm{C}$. The CGP was dissolved in $\mathrm{H}_{2} \mathrm{O}$ and precipitated again. Water-insoluble CGP was isolated from cell debris by resuspension in $0.1 \mathrm{M}$ $\mathrm{HCl}$. The now dissolved CGP was separated from insoluble material by centrifugation $(30 \mathrm{~min}, 5000 \times g)$, and it was subsequently precipitated from the supernatant by neutralization ( $\mathrm{pH} 7$ ) using 0.1 to $4 \mathrm{M} \mathrm{NaOH}$. From the supernatant of the insoluble CGP again soluble CGP was isolated. The washing steps were repeated three times for insoluble CGP to further purify the polymer. The precipitated insoluble CGP was then again sedimented by centrifugation and washed twice with $\mathrm{H}_{2} \mathrm{O}$. Persistent protein impurities were removed after the first

Table 2 Details about the used cyanophycin synthetases

\begin{tabular}{|c|c|c|c|c|}
\hline CphA & Length & Origin & CGP solubility & Reference \\
\hline $\mathrm{CphA}_{6308} \Delta 1$ & 873 AS & $\begin{array}{l}\text { CphA from Synechocystis sp. strain PCC } 6308 \text { shortened by one AS at the } \\
\text { C-terminus }\end{array}$ & Insoluble and soluble & Steinle et al. (2010) \\
\hline $\mathrm{CphA}_{6308} \Delta 1_{-}$C595S & 873 AS & $\begin{array}{l}\text { CphA from Synechocystis sp. strain PCC } 6308 \text { with point mutation and short- } \\
\text { ened by one AS at the C-terminus (see Fig. S1) }\end{array}$ & Insoluble and soluble & Steinle et al. (2010) \\
\hline CphA $_{\text {Dh }}$ & 885 AS & CphA from Desulfitobacterium hafniense DSM 10664 & Only soluble & Ziegler et al. (2002) \\
\hline
\end{tabular}


precipitation by adding $100 \mu \mathrm{g}$ proteinase $\mathrm{K}$ per $\mathrm{ml}$ and by incubating the CGP for $3 \mathrm{~h}$ at $60^{\circ} \mathrm{C}$.

\section{Analysis of isolated cyanophycin}

The purity and the molecular masses of isolated CGP were determined by sodium dodecylsulphate polyacrylamide gel electrophoresis (SDS-PAGE) in 11.5\% (wt/ vol) polyacrylamide gels, according to Laemmli (1970). The apparent molecular weight was estimated using the PageRuler Prestained Protein Ladder (Thermo Scientific).

The amino acid compositions of CGP were determined by high-performance liquid chromatography (HPLC) using a Waters B801 column $(300 \times 4 \mathrm{~mm})$ after the polymer was converted into derivatives of the constituents as described by Aboulmagd (2001b) and Steinle (2009). Precolumn ortho-phthaldialdehyde (OPA) derivatization was performed using a Smartline autosampler 3900 according to the manual (Knauer $\mathrm{GmbH}$, Berlin, Germany). A reference kit (Kollektion AS-10 from Serva Feinbiochemica, Heidelberg, Germany) was used for calibration. Ornithine and citrulline were purchased as monohydrochloride from Fluka or Merck, respectively. CGP was hydrolyzed in $6 \mathrm{M} \mathrm{HCl}(100 \mu \mathrm{l} / \mathrm{mg})$ at $95{ }^{\circ} \mathrm{C}$ overnight, neutralized, and lyophilized before measurement.

\section{Results}

\section{Cyanophycin synthesis in C. glutamicum ATCC 13032}

The CGP synthesis in the wild type of C. glutamicum was studied with three different CphAs $\left(\mathrm{CphA}_{6308} \Delta 1\right.$, $\mathrm{CphA}_{6308} \Delta 1_{-}$C595S and $\left.\mathrm{CphA}_{\mathrm{Dh}}\right)$. For CGP synthesis the strains were cultivated for $72 \mathrm{~h}$ in CGXII medium. Optimizations in cultivation conditions improved cell growth when compared to the previous study of Wiefel et al. (2019a). As a result, the cell density could be increased up to $16.6 \mathrm{~g}$ dry mass (DW)/l (see Table 3 and Additional file 1: Fig. S2). All three strains grew similarly well, but in terms of CGP content there were marked differences between the cells harboring different genes for CphAs. With $\mathrm{CphA}_{\mathrm{Dh}}$ a CGP content over $36 \%$ of the cells dry mass was achieved, which corresponds to a CGP concentration of about $6 \mathrm{~g} / \mathrm{l}$. In contrast, cells expressing $\mathrm{CphA}_{6308} \Delta 1$ and $\mathrm{CphA}_{6308} \Delta 1 \_\mathrm{C} 595 \mathrm{~S}$ synthesized

Table 3 Comparison of different C. glutamicum strains for CGP synthesis

\begin{tabular}{|c|c|c|c|c|c|c|c|c|c|}
\hline \multirow[t]{3}{*}{ Strains } & \multirow[t]{3}{*}{ DW [g/l] } & \multicolumn{8}{|c|}{ Cyanophycin } \\
\hline & & \multirow[t]{2}{*}{ Solubility } & \multirow[t]{2}{*}{ CGP content [\%DW] } & \multicolumn{6}{|c|}{ Amino acid composition[mol\%] ${ }^{*}$} \\
\hline & & & & Asp & $\operatorname{Arg}$ & Lys & Glu & Orn & Cit \\
\hline \multicolumn{10}{|l|}{ ATCC 13032} \\
\hline \multirow[t]{2}{*}{ pVWEx1::cphA $A_{6308} \Delta 1$} & $15.9 \pm 0.1$ & Insoluble & $1.9 \pm 0.5$ & 50.1 & 38.9 & 8.7 & 0.5 & 1.0 & 0.8 \\
\hline & & Soluble & $4.0 \pm 0.1$ & 51.9 & 26.9 & 10.9 & 1.2 & 6.6 & 2.5 \\
\hline \multirow[t]{2}{*}{ pVWEx1::cphA $6308 \Delta 1$ CC595S } & $15.6 \pm 0.2$ & Insoluble & $1.4 \pm 0.3$ & 54.7 & 30.0 & 13.6 & 0.3 & 0.8 & 0.6 \\
\hline & & Soluble & $8.3 \pm 2.9$ & 51.3 & 27.5 & 10.7 & 0.9 & 6.4 & 3.2 \\
\hline \multirow[t]{2}{*}{ pVWEx1::cphA $A_{D h}$} & $16.6 \pm 0.4$ & Insoluble & $0.0 \pm 0.0$ & - & - & - & - & - & - \\
\hline & & Soluble & $36.2 \pm 0.7$ & 49.3 & 40.1 & 6.9 & 2.3 & 0.6 & 0.8 \\
\hline \multicolumn{10}{|l|}{ DM1279 } \\
\hline \multirow[t]{2}{*}{ pVWEx1::cphA $A_{6308} \Delta 1$} & $11.6 \pm 0.3$ & Insoluble & $12.2 \pm 2.1$ & 52.9 & 30.3 & 16.2 & 0.2 & 0.0 & 0.4 \\
\hline & & Soluble & $2.9 \pm 0.6$ & 45.6 & 21.0 & 29.7 & 1.0 & 2.2 & 0.5 \\
\hline \multirow{2}{*}{ pVWEx1::cphA 6308 $\Delta 1$ _C595S } & $11.7 \pm 0.2$ & Insoluble & $13.1 \pm 2.3$ & 48.0 & 29.8 & 21.6 & 0.2 & 0.0 & 0.4 \\
\hline & & Soluble & $2.1 \pm 0.5$ & 42.7 & 19.8 & 33.5 & 1.0 & 2.6 & 0.4 \\
\hline \multirow[t]{2}{*}{ pVWEx1::cphA $A_{D h}$} & $14.4 \pm 0.6$ & Insoluble & $0.0 \pm 0.0$ & - & - & - & - & - & - \\
\hline & & Soluble & $24.2 \pm 1.9$ & 48.3 & 33.3 & 15.4 & 2.2 & 0.8 & 0.0 \\
\hline \multicolumn{10}{|l|}{ ORN2 $\left(P_{g d h 4}\right)$} \\
\hline \multirow[t]{2}{*}{ pVWEx1::cphA $A_{6308} \Delta 1$} & $11.6 \pm 0.2$ & Insoluble & $1.9 \pm 0.2$ & 47.6 & 42.7 & 0.0 & 0.3 & 9.1 & 0.3 \\
\hline & & Soluble & $3.2 \pm 0.7$ & 46.0 & 43.5 & 0.0 & 0.6 & 9.4 & 0.5 \\
\hline \multirow[t]{2}{*}{ pVWEx1::cphA $A_{6308} \Delta 1$ C595S } & $11.7 \pm 0.1$ & Insoluble & $2.4 \pm 0.2$ & 42.8 & 47.9 & 0.0 & 0.3 & 8.7 & 0.3 \\
\hline & & Soluble & $2.3 \pm 0.7$ & 41.7 & 44.2 & 0.0 & 0.7 & 12.6 & 0.8 \\
\hline \multirow[t]{2}{*}{ pVWEx1::cphA $A_{D h}$} & $12.9 \pm 0.3$ & Insoluble & $0.0 \pm 0.0$ & - & - & - & - & - & - \\
\hline & & Soluble & $18.6 \pm 1.6$ & 42.8 & 43.7 & 0.0 & 1.4 & 11.7 & 0.4 \\
\hline
\end{tabular}

The strains were cultivated for $72 \mathrm{~h}$ in $100 \mathrm{ml} \mathrm{CGXII} \mathrm{medium} \mathrm{in} \mathrm{a} 500 \mathrm{ml}$ Erlenmeyer flask with two baffles at $130 \mathrm{rpm}$ and $30{ }^{\circ} \mathrm{C}$. The cultures were inoculated with an $\mathrm{OD}_{600}$ of about 0.2 and after $4 \mathrm{~h}$ IPTG was added. For the ORN2( $\left.\mathrm{P}_{\text {gdh } 4}\right)$ strains an additional $15 \mathrm{mM}$ arginine was added to the medium. The cells dry mass was determined, and the contained cyanophycin was isolated and analysed. The values indicate the mean and standard deviation of at least three independent cultivations

* The values have been rounded to one decimal place 
significantly less CGP, but the CGP accumulated contained a higher amount of alternative amino acids. The point mutation $\mathrm{CphA}_{6308} \Delta 1_{-} \mathrm{C} 595 \mathrm{~S}$ led to a $4.9 \mathrm{~mol} \%$ higher lysine content in the insoluble CGP. The lysine content in the CGP of $\mathrm{CphA}_{6308} \Delta 1_{-} \mathrm{C} 595 \mathrm{~S}$ was $13.6 \mathrm{~mol} \%$ and therefore almost twice as high as that of $\mathrm{Cph}_{\mathrm{Dh}}$.

The non-biotin limiting growth conditions in this study had an impact on the composition of the synthesized CGP. The alternative amino acids ornithine and citrulline were found for the first time in the CGP accumulated in C. glutamicum. These amino acids were mainly found in the soluble CGPs accumulated in cells expressing $\mathrm{CphA}_{6308} \Delta 1$ or $\mathrm{CphA}_{6308} \Delta 1_{-} \mathrm{C} 595 \mathrm{~S}$, respectively. This CGP thus contains all the alternative amino acids known to date that are used for in vivo CGP synthesis (see Fig. 1).

\section{Cyanophycin synthesis in a C. glutamicum mutant for lysine production}

To increase the lysine content in CGP, CGP synthesis was investigated in C. glutamicum strain DM1729 (Georgi et al. 2005), which was developed for the production of lysine. Although the strains did not reach as high cell densities as the cells of C. glutamicum ATCC 13032, up to three times more lysine was incorporated into the accumulated CGP (see Table 3 and Additional file 1: Fig. S2). It is remarkable that the strain grew better with the $\mathrm{CphA}_{\mathrm{Dh}}$ and reached a higher CGP content with $24.2 \%$ of cells dry mass. The lysine content in the CGP was $15.4 \mathrm{~mol} \%$ and was therefore significantly higher than in the CGP from the cells of C. glutamicum ATCC 13032 pVWEx1::cphA $A_{\mathrm{Dh}}$. With the other two CphAs, lysine contents of up to $33.5 \mathrm{~mol} \%$ were measured in the soluble CGP, and even in the insoluble CGP higher lysine contents were found. The higher lysine concentrations in the cells of these strains resulted in a strong increase in insoluble CGP with up to $13.1 \%$ of cells dry mass at a lysine content of up to $21.6 \mathrm{~mol} \%$. It was previously thought that the higher lysine concentration would have a greater effect on soluble CGP, as high lysine is often a characteristic of soluble CGP (Frommeyer and Steinbüchel 2013). The insoluble CGP synthesised with $\mathrm{CphA}_{6308} \Delta 1$ C595S showed a $5.4 \mathrm{~mol} \%$ higher lysine content in comparison to the CGP accumulated by the single mutant cyanophycin synthetase. The content of ornithine and citrulline was lower in all these strains than in the C. glutamicum ATCC 13032 strains.

\section{Cyanophycin synthesis in a C. glutamicum mutant for ornithine production}

Corynebacterium glutamicum ORN2 $\left(\mathrm{P}_{\text {gdh }}\right)$ is a strain developed for the increased production of ornithine (Jensen et al. 2015). In this strain, genes of the arginine biosynthesis pathway were deleted to prevent the further metabolism of ornithine. As a result, the strain is auxotrophic for arginine. In a preliminary experiment it was shown that a concentration of $15 \mathrm{mM}$ arginine in the CGXII medium is a suitable concentration for CGP synthesis, as this concentration allows good growth.

The CGP content of these strains is nevertheless lower than that of the other C. glutamicum strains investigated. With these strains ornithine contents of $10 \mathrm{~mol} \%$ on average were achieved (see Table 3 and Additional file 1: Fig. S2). These results represent a slight improvement when compared to the previous results with Saccharomyces cerevisiae, where ornithine contents of only $8 \mathrm{~mol} \%$ were achieved (Steinle et al. 2009). There were only slight differences in the amino acid composition between the different isolates. The SDS-PAGE analysis showed that there are large differences in the molecular masses of the accumulated soluble and insoluble CGPs (see Fig. 2). Most of the soluble CGP exhibited molecular masses below $15 \mathrm{kDa}$ (lane 1,

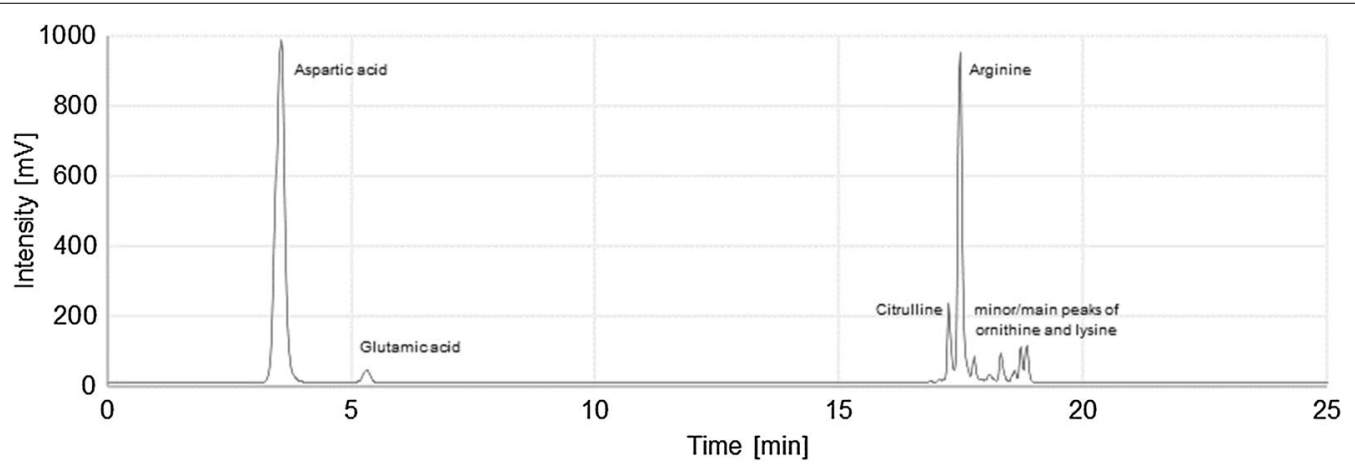

Fig. 1 HPLC chromatogram of hydrolysed soluble cyanophycin from C. glutamicum ATCC 13032 pVWEx1::cphA ${ }_{6308} \Delta 1$ _C595S with all known alternative amino acids 


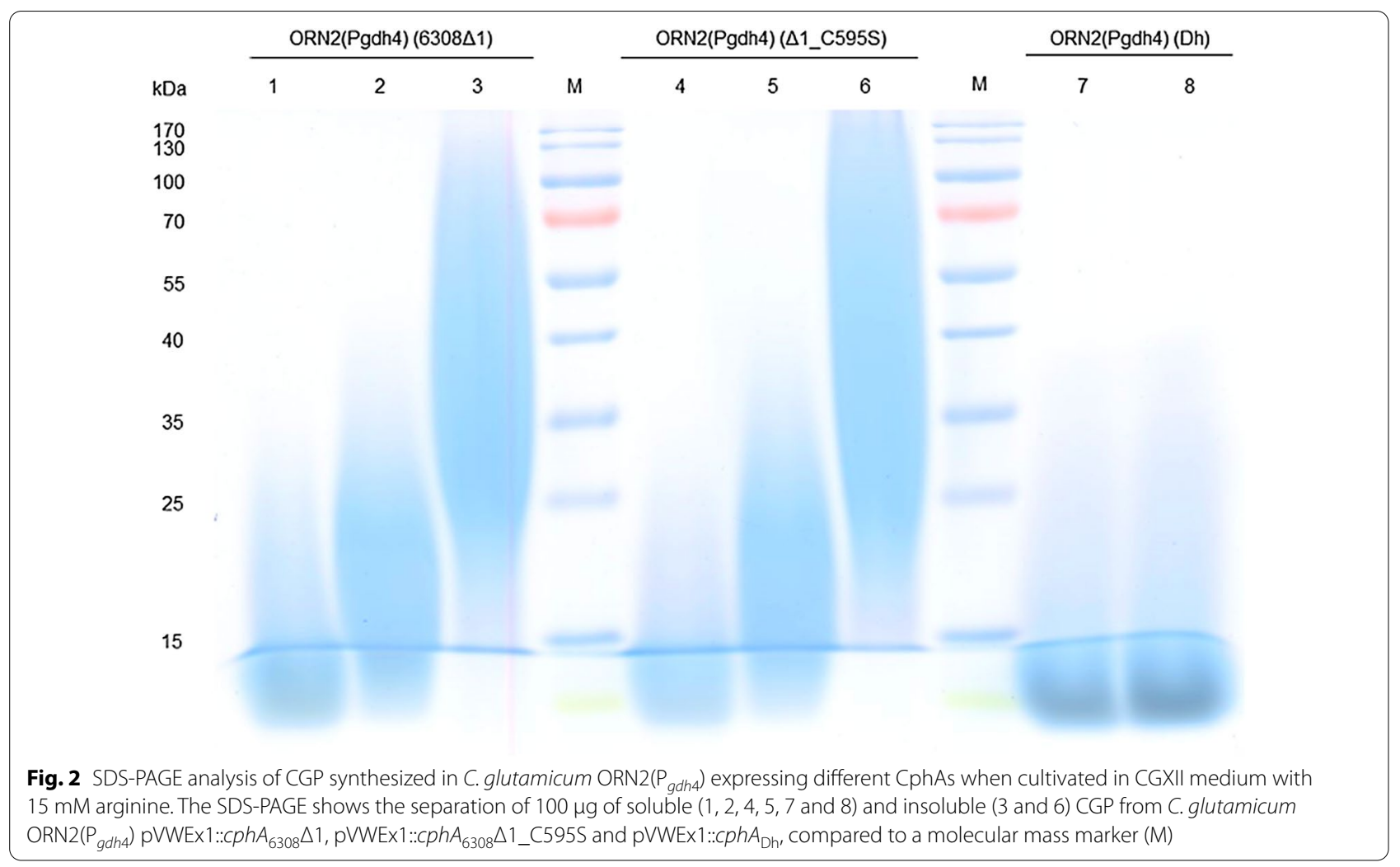

4, 7 and 8). In the case of $\mathrm{CphA}_{\mathrm{Dh}}$ it is clearly visible that most CGP has a molecular mass of only $10 \mathrm{kDa}$. The soluble CGP isolated from the supernatant of the insoluble CGP is slightly larger with 15 to $25 \mathrm{kDa}$ (lane 2 and 5). The insoluble CGP, on the other hand, exhibited a molecular weight of 35 to $55 \mathrm{kDa}$ (lane 3 and 6).

\section{Cyanophycin synthesis in a C. glutamicum mutant for cadaverine production}

In a previous in vitro study, it was shown that agmatine (biogenic amine of arginine) has a negative effect on the incorporation of arginine into the polymer. Therefore, it was suspected that it could be incorporated into CGP instead of arginine (Aboulmagd 2001a). Cadaverine is also a biogenic amine and is produced by decarboxylation of lysine. To enable the synthesis of cadaverine in C. glutamicum, the lysine decarboxylase LdcC was additionally introduced into the DM1729 strains using the vector pEC-XT99A (Sgobba et al. 2018). This is the first study on the effect of cadaverine on CGP synthesis. The conversion of lysine to cadaverine results in a significant decrease in the lysine content of CGP (see Table 4 and Additional file 1: Fig. S2). The CGP content in the cells also decreases in comparison to the DM1729 strains not harboring pEC-XT99A::ldcC.
Noticeable was the slower growth of the strain expressing $\mathrm{CphA}_{\mathrm{Dh}}$. Cadaverine could not be detected in any of the extracted CGP. Possibly cadaverine has an inhibiting effect on CGP synthesis.

\section{Cultivation in stirred tank reactors and scale up}

In the first fermentation of C. glutamicum DM1729 pVWEx1::cphA $A_{6308} \Delta 1_{-}$C595S in CGXII medium CGP biosynthesis was induced with $0.1 \mathrm{mM}$ IPTG after a cultivation period of $4 \mathrm{~h}$. The fermentation took $53 \mathrm{~h}$ in total. A maximum cells dry mass of $11 \mathrm{~g} / \mathrm{l}$ was obtained. Only insoluble CGP could be isolated. This reached an average of $2.5 \%$ of the cells dry mass. The lysine content was $16.7 \mathrm{~mol} \%$.

In the second fermentation with addition of $5 \mathrm{~g} / \mathrm{l}$ CASO broth, $0.1 \mathrm{mM}$ IPTG was used directly at the start of the fermentation to induce cells for production of CGP. Based on the $\mathrm{OD}_{600}$, it could be seen that the cells grew significantly faster and they reached a higher cells dry mass of $15 \mathrm{~g} / \mathrm{l}$ than in the flask. Although soluble and insoluble CGP could be isolated, the total CGP content was again only $2.5 \%$ of the cells dry mass. The amino acid composition was comparable to that of the CGP formed in the flask experiment. 
Table 4 Comparison of CGP synthesis in C. glutamicum strains for cadaverine production

\begin{tabular}{|c|c|c|c|c|c|c|c|c|c|c|}
\hline \multirow[t]{3}{*}{ Strains } & \multirow[t]{3}{*}{$\mathrm{DW}[\mathrm{g} / \mathrm{l}]$} & \multicolumn{9}{|c|}{ Cyanophycin } \\
\hline & & \multirow[t]{2}{*}{ Solubility } & \multirow[t]{2}{*}{ CGP content [\%DW] } & \multicolumn{7}{|c|}{ Amino acid composition [mol\%] * } \\
\hline & & & & $\overline{A s p}$ & $\operatorname{Arg}$ & Lys & Glu & Orn & Cit & $\mathrm{Cad}$ \\
\hline \multicolumn{11}{|l|}{ DM1279 pEC-XT99A::IdcC } \\
\hline \multirow[t]{2}{*}{ pVWEx1::cphA $A_{6308} \Delta 1$} & $13.3 \pm 0.1$ & Insoluble & $3.5 \pm 0.9$ & 46.6 & 46.2 & 6.2 & 0.4 & 0.1 & 0.5 & 0.0 \\
\hline & & Soluble & $3.5 \pm 1.4$ & 44.1 & 38.0 & 13.6 & 1.6 & 2.2 & 0.5 & 0.0 \\
\hline \multirow[t]{2}{*}{ pVWEx1::cphA $A_{6308} \Delta 1$ _C595S } & $12.7 \pm 0.3$ & Insoluble & $3.2 \pm 1.2$ & 47.4 & 45.8 & 5.9 & 0.4 & 0.1 & 0.4 & 0.0 \\
\hline & & Soluble & $1.5 \pm 1.2$ & 44.2 & 37.1 & 16.0 & 1.9 & 0.0 & 0.8 & 0.0 \\
\hline \multirow[t]{2}{*}{ pVWEx1::cphA $A_{D h}$} & $9.2 \pm 0.1$ & Insoluble & $0.0 \pm 0.0$ & - & - & - & - & - & - & - \\
\hline & & Soluble & $20.4 \pm 0.7$ & 44.1 & 41.9 & 11.0 & 2.2 & 0.5 & 0.3 & 0.0 \\
\hline
\end{tabular}

The strains were cultivated for $72 \mathrm{~h}$ in $100 \mathrm{ml} \mathrm{CGXII} \mathrm{medium} \mathrm{in} 500 \mathrm{ml}$ Erlenmeyer flasks with two baffles at $130 \mathrm{rpm}$ and $30{ }^{\circ} \mathrm{C}$. The cultures were inoculated with an $\mathrm{OD}_{600}$ of about 0.2 and after $4 \mathrm{~h}$ IPTG was added. The cells dry mass was determined and the contained cyanophycin was isolated and analyzed. The values indicate the mean and standard deviation of at least three independent cultivations

* The values have been rounded to one decimal place

\section{Discussion}

Based on the study of Wiefel et al. (2019a), the cultivation conditions were further optimized to improve cell growth and to increase CGP yield. The omission of biotin limitation had the greatest impact, even if this means that less glutamic acid is incorporated into CGP. The growth of the wild type strains could thus be increased to a cells dry mass of $16 \mathrm{~g} / \mathrm{l}$, and a CGP content of $36.2 \%$ of the cells dry mass was achieved with $\mathrm{CphA}_{\mathrm{Dh}}$. In comparison, the cultivation of C. glutamicum ATCC 13032 pVWEx1::cph $A_{\mathrm{Dh}}$ with biotin limitation in Wiefel et al. (2019a) achieved only a CGP content of $17.1 \%$ of cells dry mass. For the CphA variants of Synechocystis $s p$. PCC6308 it was possible for the first time to isolate water soluble CGP from cells cultivated in CGXII medium. The CGP contents of cells harboring these CphAs were significantly lower than that of cells harboring $\mathrm{CphA}_{\mathrm{Dh}}$. However, due to the soluble CGP, a slight increase compared to previous studies (Aboulmagd et al. 2001b; Wiefel et al. 2019a) was observed. Due to the changes in cultivation conditions, ornithine and citrulline could be detected in CGP from C. glutamicum for the first time. However, the amounts of citrulline were very low. With S. cerevisiae, for example, citrulline contents of $20 \mathrm{~mol} \%$ have been already achieved (Steinle et al. 2009).

Experiments with the mutants of C. glutamicum showed that the proportion of alternative amino acids in CGP can be increased in these strains. The lysine contents were more than doubled by DM1729 in many samples and much more insoluble CGP was synthesized. This could possibly be related to the intracellular $\mathrm{pH}$ increasing due to the higher lysine concentration and thus being closer to the optimal $\mathrm{pH}$ of 8.2 of $\mathrm{CphA}_{6308}$ (Aboulmagd et al. 2001a). It is noticeable that due to the point mutation C595S in the insoluble CGP the lysine content is $5.4 \mathrm{~mol} \%$ higher. Also in $P$. pastoris and E. coli the lysine contents were slightly higher if the point mutation C595S of CphA was used (Steinle et al. 2010). However, the increase was not as high as in this case. This effect has already been observed with C. glutamicum ATCC 13032. The point mutation seems to have a positive influence on the incorporation of lysine.

An increase of the alternative amino acid was also observed in the ornithine-forming strains. Here, a maximum ornithine content of $12.6 \mathrm{~mol} \%$ was reached. The proportion is thus higher than in the study of Steinle et al. 2009, where with S. cerevisiae an ornithine proportion of $8 \mathrm{~mol} \%$ was achieved. The differences in the composition of soluble and insoluble CGP were very small in these strains, which suggests that the size of the polymer also has an influence on the solubility behavior, as this was the main difference. This assumption was already made in Wiefel et al. (2019a).

The decarboxylation of lysine to cadaverine has resulted in a lower cellular cyanophycin content and thus in less CGP synthesised and in a drastic reduction in the lysine content of the polymer. However, no cadaverine could be detected in cyanophycin. The strains for cadaverine production have therefore proved unsuitable for cyanophycin synthesis.

Although the growth of the mutants is lower than that of C. glutamicum ATCC 13032, mutants that produce more lysine in particular have shown great potential for CGP synthesis. It would therefore be useful to investigate this further and to optimize the scale up, because until now growth has been improved on a larger scale, but the CGP content is lower than in flasks. 


\begin{abstract}
Abbreviations
Arg: Arginine; AS: Amino acids; Asp: Aspartic acid; Cad: Cadaverine; CGP: Cyanophycin grana peptide; Cit: Citrulline; CphA: Cyanophycin synthetases; CphB: Intracellular cyanophycinase; CphE: Extracellular cyanophycinase; DW: Dry weight; Glu: Glutamic acid; LB: Lysogeny broth; Lys: Lysine; $\mathrm{OD}_{600}$ : Optical density at $600 \mathrm{~nm}$; OPA: ortho-Phthaldialdehyde; Orn: Ornithine; RBS: Ribosome binding site; WT: Wild type.
\end{abstract}

\section{Supplementary Information}

The online version contains supplementary material available at https://doi. org/10.1186/s13568-021-01217-5.

Additional file 1.: Fig. S1. Sequence alignment of $c p h A_{6308}$ and $c p h A_{6308} \Delta 1 \_C 595 S$. Fig. S2. Graphical representation of the results obtained with the different C. glutamicum strains for CGP synthesis.

\section{Authors' contributions}

RW carried out this study and drafted the manuscript. LW and AS supervised the study and revised the manuscript. VFW provided strains and helped with molecular genetic methods. All authors read and approved the final manuscript.

\section{Funding}

No funding was received.

\section{Availability of data and materials}

All datasets on which the conclusions of the manuscript are presented in the main paper.

\section{Declarations}

Ethics approval and consent to participate

Not applicable.

\section{Consent for publication}

Not applicable.

\section{Competing interests}

On behalf of all authors, the corresponding author states that there is no conflict of interest.

\section{Author details}

${ }^{1}$ Institut für Molekulare Mikrobiologie und Biotechnologie (IMMB), Westfälische Wilhelms-Universität Münster, Corrensstraße 3, 48149 Münster, Germany. ${ }^{2}$ Faculty of Biology \& CeBiTec, Bielefeld University, Universitätsstraße 25, 33615 Bielefeld, Germany. ${ }^{3}$ Environmental Science Department, King Abdulaziz University, Jeddah, Saudi Arabia.

Received: 31 January 2021 Accepted: 7 April 2021

Published online: 15 April 2021

\section{References}

Aboulmagd E, Oppermann-Sanio FB, Steinbüchel A (2001a) Purification of Synechocystis sp. strain PCC6308 cyanophycin synthetase and its characterization with respect to substrate and primer specificity. Appl Environ Microbiol 67:2176-2182. https://doi.org/10.1128/AEM.67.5.2176-2182. 2001

Aboulmagd E, Voss I, Oppermann-Sanio FB, Steinbüchel A (2001b) Heterologous expression of cyanophycin synthetase and cyanophycin synthesis in the industrial relevant bacteria Corynebacterium glutamicum and Ralstonia eutropha and in Pseudomonas putida. Biomacromol 2:1338-1342. https://doi.org/10.1021/bm010075a

Berg H, Ziegler K, Piotukh K, Baier K, Lockau W, Volkmer-Engert R (2000) Biosynthesis of the cyanobacterial reserve polymer multi-L-arginyl-poly- $L$ aspartic acid (cyanophycin). Eur J Biochem 267:5561-5570. https://doi. org/10.1046/j.1432-1327.2000.01622.x

Frommeyer M, Steinbüchel A (2013) Increased lysine content is the main characteristic of the soluble form of the polyamide cyanophycin synthesized by recombinant Escherichia coli. Appl Environ Microbiol 79:4474-4483. https://doi.org/10.1128/AEM.00986-13

Frommeyer M, Bergander K, Steinbüchel A (2014) Guanidination of soluble lysine-rich cyanophycin yields a homoarginine-containing polyamide. Appl Environ Microbiol 80:2381-2389. https://doi.org/10.1128/AEM. 04013-13

Georgi T, Rittmann D, Wendisch VF (2005) Lysine and glutamate production by Corynebacterium glutamicum on glucose, fructose and sucrose. Roles of malic enzyme and fructose-1,6-bisphosphatase. Metab Eng 7:291-301. https://doi.org/10.1016/j.ymben.2005.05.001

Jensen JVK, Eberhardt D, Wendisch VF (2015) Modular pathway engineering of Corynebacterium glutamicum for production of the glutamate-derived compounds ornithine, proline, putrescine, citrulline, and arginine. J Biotechnol 214:85-94. https://doi.org/10.1016/j.jbiotec.2015.09.017

Keilhauer C, Eggeling L, Sahm H (1993) Isoleucine Synthesis in Corynebacterium glutamicum: molecular analysis of the ilvB-ilvN-ilvC operon. J Bacteriol 175:5595-5603. https://doi.org/10.1128/jb.175.17.5595-5603.1993

Laemmli UK (1970) Cleavage of structural proteins during the assembly of the head of bacteriophage T4. Nature 227:680-685. https://doi.org/10.1038/ $227680 \mathrm{a} 0$

Persicke M, Plassmeier J, Neuweger H, Rückert C, Pühler A, Kalinowski J (2011) Size exclusion chromatography. An improved method to harvest Corynebacterium glutamicum cells for the analysis of cytosolic metabolites. J Biotechnol 154:171-178. https://doi.org/10.1016/j.jbiotec.2010.08. 016

Peters-Wendisch PG, Schiel B, Wendisch VF, Katsoulidis E, Möckel B, Sahm $\mathrm{H}$, Eikmanns BJ (2001) Pyruvate carboxylase is a major bottleneck for glutamate and lysine production by Corynebacterium glutamicum. J Mol Microbiol Biotechnol 3:295-300

Sallam A, Kast A, Przybilla S, Meiswinkel T, Steinbüchel A (2009) Biotechnological process for production of beta-dipeptides from cyanophycin on a technical scale and its optimization. Appl Environ Microbiol 75:29-38. https://doi.org/10.1128/AEM.01344-08

Sallam A, Steinbüchel A (2010) Dipeptides in nutrition and therapy. Cyanophycin-derived dipeptides as natural alternatives and their biotechnological production. Appl Microbiol Biotechnol 87:815-828. https://doi.org/10. 1007/s00253-010-2641-0

Sambrook J, Fritsch E, Maniatis T (1989) Molecular cloning: a laboratory manual, 2nd edn. Cold Spring Harbor Laboratory Press, New York

Sgobba E, Blöbaum L, Wendisch VF (2018) Production of food and feed additives from non-food-competing feedstocks. Valorizing $\mathrm{N}$-acetylmuramic acid for amino acid and carotenoid fermentation with Corynebacterium glutamicum. Front Microbiol 9:2046. https://doi.org/10.3389/fmicb.2018. 02046

Siebert D, Wendisch VF (2015) Metabolic pathway engineering for production of 1,2-propanediol and 1-propanol by Corynebacterium glutamicum. Biotechnol Biofuels 8:91. https://doi.org/10.1186/s13068-015-0269-0

Steinle A, Bergander K, Steinbüchel A (2009) Metabolic engineering of Saccharomyces cerevisiae for production of novel cyanophycins with an extended range of constituent amino acids. Appl Environ Microbiol 75:3437-3446. https://doi.org/10.1128/AEM.00383-09

Steinle A, Witthoff S, Krause JP, Steinbüchel A (2010) Establishment of cyanophycin biosynthesis in Pichia pastoris and optimization by use of engineered cyanophycin synthetases. Appl Environ Microbiol 76:1062-1070. https://doi.org/10.1128/AEM.01659-09

Tauch A, Kirchner O, Löffler B, Götker S, Pühler A, Kalinowski J (2002) Efficient electrotransformation of Corynebacterium diphtheriae with a mini-replicon derived from the Corynebacterium glutamicum plasmid pGA1. Curr Microbiol 45:362-367. https://doi.org/10.1007/s00284-002-3728-3

van der Rest ME, Lange C, Molenaar D (1999) A heat shock following electroporation induces highly efficient transformation of Corynebacterium glutamicum with xenogeneic plasmid DNA. Appl Microbiol Biotechnol 52:541-545. https://doi.org/10.1007/s002530051557

Wendisch VF (2020) Metabolic Engineering advances and prospects for amino acid production. Metab Eng 58:17-34. https://doi.org/10.1016/j.ymben. 2019.03.008 
Wiefel L, Bröker A, Steinbüchel A (2011) Synthesis of a citrulline-rich cyanophycin by use of Pseudomonas putida ATCC 4359. Appl Microbiol Biotechnol 90:1755-1762. https://doi.org/10.1007/s00253-011-3224-4

Wiefel L, Steinbüchel A (2014) Solubility behavior of cyanophycin depending on lysine content. Appl Environ Microbiol 80:1091-1096. https://doi.org/ 10.1128/AEM.03159-13

Wiefel L, Steinbüchel A (2016) Enzymatic modification of soluble cyanophycin using the type II peptidyl arginine deiminase from Oryctolagus cuniculus. Macromol Biosci 16:1064-1071. https://doi.org/10.1002/mabi.201500433

Wiefel L, Wohlers K, Steinbüchel A (2019a) Re-evaluation of cyanophycin synthesis in Corynebacterium glutamicum and incorporation of glutamic acid and lysine into the polymer. Appl Microbiol Biotechnol 103:4033-4043. https://doi.org/10.1007/s00253-019-09780-5

Wiefel L, Bachmann F, Terwort J, Steinbüchel A (2019b) In vitro modification of bacterial cyanophycin and cyanophycin dipeptides using chemical agents towards novel variants of the biopolymer. Earth Syst Environ 3:637-650. https://doi.org/10.1007/s41748-019-00107-y

Ziegler K, Deutzmann R, Lockau W (2002) Cyanophycin synthetase-like enzymes of non-cyanobacterial eubacteria: characterization of the polymer produced by a recombinant synthetase of Desulfitobacterium hafniense. Z Naturforsch 57:522-529. https://doi.org/10.1515/ znc-2002-5-62

\section{Publisher's Note}

Springer Nature remains neutral with regard to jurisdictional claims in published maps and institutional affiliations.

\section{Submit your manuscript to a SpringerOpen ${ }^{\circ}$ journal and benefit from:}

- Convenient online submission

- Rigorous peer review

- Open access: articles freely available online

- High visibility within the field

- Retaining the copyright to your article

Submit your next manuscript at $\boldsymbol{s p r i n g e r o p e n . c o m ~}$ 